\title{
Convection-pulsation interaction: theories and applications
}

\author{
M.-A. Dupret ${ }^{1}$, A. Grigahcène ${ }^{2,3}$, M. Gabriel $^{4}$, R. Garrido ${ }^{2}$ \\ ${ }^{1}$ Observatoire de Paris, LESIA, CNRS UMR 8109, 92195 Meudon, France \\ ${ }^{2}$ Instituto de Astrofísica de Andalucía-CSIC, Apartado 3004, 18080 Granada, Spain \\ ${ }^{3}$ CRAAG - Algiers Observatory BP 63 Bouzareah 16340, Algiers, Algeria \\ ${ }^{4}$ Institut d'Astrophysique et de Géophysique de I'Université de Liège, Belgium
}

\begin{abstract}
The problem of the interaction between convection and pulsations is considered. A precise modeling of this interaction is required for the seismic study of stars with non-negligible convective envelope. After a short introduction to the Time-Dependent Convection theory of Gabriel (1996) and Grigahcène et al. (2005), we give a summary of the main results obtained with this treatment for $\delta$ Sct, $\gamma$ Dor and solar-like stars.
\end{abstract}

\section{Introduction}

All stars with $T_{\text {eff }} \lesssim 8500 \mathrm{~K}$ have a superficial convective zone. Many of these stars are pulsating and taking the interaction with convection into account is very important for their seismic analysis. A first important aspect is the analysis of the driving and damping mechanisms at the origin of the oscillations. The transition region where the thermal relaxation time is of the same order as the pulsation period plays the major role in theses mechanisms. This region is located inside the superficial convective zone in cold $\delta$ Sct stars, in $\gamma$ Dor stars and in solar-like stars. Hence, the convection-pulsation interaction plays a central role in the driving and damping mechanisms. A second important aspect is the analysis of the multi-color amplitudes and phases and their use for mode identification. These observables are mainly determined by the energy transfer associated to the oscillations in the upper part of the convective envelope, so that the convection-pulsation interaction plays also a central role at this level.

\section{Time-Dependent Convection (TDC) theories}

There are different approaches for the study of the convection-pulsation interaction. First, 3D hydrodynamical simulations can be performed. All motions, and thus in particular the acoustic modes are present in the turbulence spectrum. Hence, the analysis of such simulations would enable us to study the energetics of the oscillations inside a convective zone. However, in addition to the fact that these simulations are highly computer-time consuming, present simulations (Nordlund \& Stein 2000) are restricted to the thin upper part of the convection zone and they are only able to give the uncoherent effect of the convection on the oscillations. This can be used to determine the power injected in the acoustic modes for stochastically excited oscillators (Samadi et al. 2003). But the damping rates of the modes in solar-like oscillators or their growth rates in auto-driven oscillators $(\delta$ Sct, $\gamma$ Dor stars, $\ldots)$ as well as the amplitude and phases cannot be determined by this approach. 
The only present way to determine these quantities is the perturbative approach. In this approach, the acoustic and convective motions are separated by assuming that the first have much larger wavelengths than the last ones in the spectrum of turbulence. Different perturbative Time-Dependent Convection (TDC) theories have been derived. The theories of Gough (1977) and Gabriel (1996) are based on the Mixing-Length (ML) approach, while the theory of Xiong (1998) is more sophisticated. The theory of Gabriel (1996) is the only one valid for non-radial modes and we consider it with more details here. The general methodology of this theory is the following, we refer to Grigahcène et al. (2005) for more details. As usually in the study of turbulence, the different physical quantities are separated into a mean part and a convective fluctuation part. We obtain then hydrodynamic equations for the average medium and for the convective fluctuations. The perturbation of the mean equations gives the linear equations for the non-adiabatic oscillations we have to solve. But in these equations, appear unknown perturbed correlation terms: the perturbation of the convective flux, of the Reynolds stress tensor and of the dissipation rate of turbulent kinetic energy into heat. These terms are obtained by perturbing the equations for the convective fluctuations. In order to have as much equations as variables, it is necessary to add some closure equations. In the mixing-length approach, the problem is closed immediately by introducing an ad-hoc life-time of the convective elements $\tau_{\mathrm{c}}$, which leads to the following movement and energy equations for the convective fluctuations:

$$
\begin{gathered}
\bar{\rho} \frac{\mathrm{d} \vec{V}}{\mathrm{~d} t}=\frac{\Delta \rho}{\bar{\rho}} \nabla \bar{p}-\nabla \Delta p-\rho \vec{V} \cdot \nabla \vec{u}-\frac{8}{3} \frac{\bar{\rho} \vec{V}}{\tau_{\mathrm{c}}}, \\
\frac{\Delta(\rho T)}{\overline{\rho T}} \frac{\mathrm{d} \bar{s}}{\mathrm{~d} t}+\frac{\mathrm{d} \Delta s}{\mathrm{~d} t}+\vec{V} \cdot \nabla \bar{s}=-\frac{\omega_{\mathrm{R}} \tau_{\mathrm{c}}+1}{\tau_{\mathrm{c}}} \Delta s .
\end{gathered}
$$

In the stationnary case, this leads to the classical MLT equations adopted in most of the stellar evolution codes. The things are, however, more complex when we consider the perturbation of these equations. The problem is that all the turbulence cascade is hidden behind the closure terms $\bar{\rho} \vec{V} / \tau_{\mathrm{c}}$ and $\Delta s / \tau_{\mathrm{c}}$, and it is not known how this cascade adapts to the oscillations. The simplest way is to assume $\delta \tau_{\mathrm{c}} / \tau_{\mathrm{c}}=\delta V_{r} / V_{r}-\delta l / l=\delta V_{r} / V_{r}-\delta H_{p} / H_{p}$. We will refer later to other possibilities. Finally, we search for solutions of the perturbed equations for the convection in the form of plane waves: $\delta(\Delta X)=\delta(\Delta X)_{\vec{k}} e^{i \vec{k} \cdot \vec{r}} e^{i \sigma t}$. Taking the appropriate means, the perturbed convective flux, Reynolds stress tensor and dissipation rate of turbulent kinetic energy can be expressed in terms of the usual perturbed mean quantities $\delta \bar{s}, \delta \bar{\rho}, \delta \bar{P}, \delta \vec{r} \ldots$ These equations are substituted in the general system for the linear nonadiabatic stellar oscillations. And finally, implementing this set of equations in a non-adiabatic pulsation code, we can solve this system. The solutions give the frequencies and damping rates or growth rates (eigenvalues) and the normalized amplitudes and phases of variation of the different physical quantities (eigenfunctions) for the different modes and for the considered stellar model.

\section{Problems associated with TDC treatments}

A well known problem associated with local TDC treatments is the occurrence of shortwavelength spatial oscillations of the thermal eigenfunctions in the efficient part of the convective envelope (where $\sigma \tau_{\mathrm{c}} \gg 1$ ), which is in contradiction with the basic assumptions of the MLT; it occurs mainly in solar-type oscillators. As shown in Grigahcène et al. (2005), this problem is directly linked to the treatment adopted for the perturbation of the energy closure equation. These authors proposed a new perturbation of this equation:

$$
\delta\left(\frac{\Delta s}{\tau_{\mathrm{C}}}\right)=\frac{\Delta s}{\tau_{\mathrm{C}}}\left(\left(1+\beta \sigma \tau_{\mathrm{c}}\right) \frac{\delta \Delta s}{\Delta s}-\frac{\delta \tau_{\mathrm{C}}}{\tau_{\mathrm{C}}}\right),
$$


where a new free parameter $\beta$ was introduced. This new parameter allows us to take into account that the characteristic time of the perturbation of the dissipations in the energy equation (2) is the minimum of $\tau_{\mathrm{c}}$ and of the period $\mathrm{P}$ (and not always $\tau_{\mathrm{c}}$ ). A complex value of $\beta$ can also try to mimic the phase shift produced by the energy cascade. Thanks to the real part of this parameter, the oscillatory form of the energy and transfer perturbed equations disappears, which solves the problem of the short-wavelength spatial oscillations. Another problem is the non-local character of turbulence. This problem is considered in details in our other paper of these proceedings. We note that non-local treatments allow also to avoid the problem of the short-wavelength spatial oscillations.

\section{4. $\delta$ Scuti stars}

We consider now the application of TDC models to $\delta$ Sct stars. A first important result is that TDC models succeed in stabilizing the $\delta$ Sct-type low order p-modes at the red border of the instability strip. This result was obtained by different authors, for radial modes (Houdek 2000, Xiong et al. 2001) and for non-radial modes by Dupret et al. (2004a, 2005a) and Grigahcène et al. (2004). On the contrary, it is impossible to obtain the stabilization of the modes with Frozen Convection (FC) models. However, the physical mechanism at the origin of this stabilization remains a matter of debate. In the models of Houdek (2000), the main damping is due to the turbulent pressure variations $\left(\delta p_{t}\right)$. But to the opposite, $\delta p_{t}$ has a driving effect in the models of Xiong et al. (2001). In the models used by Dupret et al. (2005a) (Gabriel's TDC theory), the turbulent pressure variations and the dissipation rate of turbulent kinetic energy variations have the opposite effect on the driving and damping and their combined effect is small. In these models, the main damping is due to the effect of convective flux variations, which cancels the flux blocking driving at the base of the Convective Envelope (CE) and the $\kappa$-mechanisms in the $\mathrm{He}$ and $\mathrm{H}$ partial ionization zones. We emphasize that, in order to have the stabilization of the modes at the observed red border of the $\delta$ Sct instability strip, it is necessary that the CE of these models goes down deep enough, including the $\mathrm{He}$ and $\mathrm{H}$ partial ionization zones. In the frame of the MLT, this can be achieved only with $\alpha \gtrsim 1.8$ (solar calibrated value).

Another very important aspect is the analysis of the multi-color photometric amplitude ratios and phase differences and their use for mode identification. Dupret et al. (2005c) were the first to use TDC models in this frame. At the blue side of the $\delta$ Sct instability strip $\left(T_{\text {eff }} \gtrsim 7500 \mathrm{~K}\right)$, the superficial convective zone is very thin, so that TDC and FC results are similar. But it is the contrary at the red side of the instability strip. The main differences between TDC and FC results are in the phase-lag $\psi_{T}$ (phase difference between the effective temperature variation and the radial displacement). In FC models, $\psi_{T}$ goes down towards $0^{\circ}$ at low effective temperature, while in TDC ones, $\psi_{T}$ remain near $90^{\circ}$. Comparison with observations shows that TDC results agree much better with observations than FC ones (Dupret et al. 2005c). This allows a much more secure identification of the degree $\ell$ in $\delta$ Sct stars.

\section{5. $\gamma$ Doradus stars}

We consider now the case of $\gamma$ Dor stars. A first important result concerns the driving of their high-order gravity modes. Guzik et al. (2000) showed that periodic flux blocking at the base of the CE can explain this driving. They used FC models, which is acceptable in a thin region near the CE bottom, but not in the rest of the CE where the life-time of the convective elements is smaller than the pulsation periods. It is thus important to use TDC models to describe correctly this driving. This was done by Dupret et al. (2004a, 2005a) who showed that the flux blocking mechanism survives when TDC is considered. We emphasize that, in 
order to have a theoretical instability strip in agreement with observations, a deep enough CE is required with its bottom in the transition region for g-modes. In the frame of the MLT, this can be achieved only with $\alpha \simeq 2$.

Secondly, the use of TDC models is very important for the analysis of the amplitude ratios and phase differences. As shown by Dupret et al. (2005b), the phase-lags $\psi_{T}$ predicted by TDC models (near $0^{\circ}$ ) agree well with the observed spectro-photometric phase differences, contrary to the FC ones which are near $180^{\circ}$ for high $\alpha$. The multi-color photometric amplitude ratios predicted by TDC models also better agree with observations than the FC ones. TDC models are thus required for the photometric identification of the degree $\ell$ in $\gamma$ Dor stars.

\section{Solar-like stars}

Finally, we consider the application to solar-like stars. These stars are stable oscillators whose excitation is due to stochastic forcing by turbulent motions. The non-adiabatic interaction between convection and oscillations is particularly difficult to model in these stars, as shown above. The interesting point is that strong constraints on this interaction can be obtained for these stars. First, the theoretical damping rates which are very sensitive to the details of the TDC treatment can be compared with the observations given by the line-widths in the power spectrum. Secondly, the theoretical amplitudes can be obtained from the ratio between the power injected stochastically in the acoustic modes (Samadi et al. 2003) and their damping rate, and they can also be compared with observations. The Sun gives the strongest constraints on these observables and it is difficult to obtain TDC models agreeing with them. Encouraging results have been obtained by Balmforth (1992) and Dupret et al. (2004b).

Acknowledgments. MAD acknowledges financial support by CNES.

\section{References}

Balmforth, N. J. 1992, MNRAS, 255, 603

Dupret, M.-A., Grigahcène, A., Garrido, R., et al. 2004a, A\&A 414, L17

Dupret, M.-A., Grigahcène, A., Garrido, R., et al. 2004b, In: SOHO 14 / GONG 2004 Workshop (ESA SP-559), New Haven, eds. D. Danesy., p. 207

Dupret, M.-A., Grigahcène, A., Garrido, R., et al. 2005a, A\&A 435, 927

Dupret, M.-A., Grigahcène, A., Garrido, R., et al. 2005b, MNRAS 360, 1143

Dupret, M.-A., Grigahcène, A., Garrido, R., et al. 2005c, MNRAS 361, 476

Gabriel, M. 1996, Bull. Astron. Soc. of India 24, 233

Gough, D. O. 1977, ApJ 214, 196

Grigahcène, A., Dupret, M.-A., Garrido, R., et al. 2004, in "JENAM Minisymposium in Asteroseismology", eds. Z. Kollath, G. Handler, CoAst 145, 9

Grigahcène, A., Dupret, M.-A., Gabriel, M., et al. 2005, A\&A 434, 1055

Guzik, J. A., Kaye, A. B., Bradley, P. A., et al. 2000, ApJ 542, L57

Houdek, G. 2000, in "The 6th Vienna Workshop on $\delta$ Scuti and Related Stars, eds. M. Montgommery, M. Breger, ASP Conf. Ser. 210, 454

Nordlund, A., \& Stein, R. F. 2001, ApJ 546, 576

Samadi, R., Nordlund, A., Stein, R., et al. 2003, A\&A 404, 1129

Xiong, D. R., Cheng, Q. L., \& Deng, L. 1997, ApJS 108, 529

Xiong, D. R., \& Deng, L. 2001, MNRAS 324, 243 